\title{
Des documents numériques aux « dépôts » numériques
}

\author{
Michel Chabin \\ Archive 17 \\ 20, rue Gassion \\ F-64000 PAU \\ michel.chabin@wanadoo.fr
}

\begin{abstract}
RÉSUMÉ. Au cours des derniers siècles, le réseau des dépôts d'archives s'est structuré sous l'effet des contraintes liées aux caractères physiques des supports des documents : leur poids, leur nombre, leur encombrement. Les archives devaient quitter tôt ou tard le lieu de leur production, mais pour se déposer à proximité, d'où le maillage territorial des dépôts. L'avènement du numérique change la donne. Les anciennes contraintes disparaissent. Des tensions nouvelles vont ébranler le système d'organisation des archives et modifier profondément son organisation. Notamment les contraintes économiques contradictoires des producteurs et utilisateurs d'archives. L'arbitrage qui sera rendu dessinera le nouveau réseau des "dépôts numériques".
\end{abstract}

ABSTRACT. During the last centuries, the archival repositories network has been organised in a way linked to the physical characteristics of the media: weight, number or mass. Early or late, the records had to be transferred from their place of creation to an archival institution, usually near by: this is the reason of our territorial organisation of the archives, from local to national repositories. With information technology, the situation is no more the same. Old constraints are disappearing. New issues are going to shake this archival network and deeply modify the way it is organised. In particular, economical requirements of the creator don't fit with the needs of the users. The decision taken to face these issues will draw a new network of "digital repositories".

MOTS-CLÉS: archives, réseau archivistique, territorialité, dépôt numérique, archiviste, archivistique, archivistique spéciale.

KEY WORDS: archives, archival network, territoriality, digital repository, archivist, archival science.

Document numérique. Volume 4 -n 3-4/2000, pages 301 à 314 


\section{Introduction}

L'époque, pas si lointaine, où les documents numériques étaient regardés comme des curiosités technologiques porteuses d'avenir, sinon comme des trublions qu'il importait de faire rentrer dans le rang, est aujourd'hui bien révolue. Ils sont partout, ils se sont banalisés. Souvent moins chers et plus efficaces, ils sont de redoutables concurrents pour les documents traditionnels, accélérant ici leur disparition, interrompant là leurs cycles de production, les confinant ailleurs dans des réserves ou des emplois spécifiques et marginaux. Au point que l'on est en droit de se demander si la requête unanime des archivistes et autres gestionnaires de documents de disposer d'urgence, pour les mieux gérer, de normes, de procédures et de règles standardisées, ouvertes et partagées n'est pas déjà, sans être inutile, fondamentalement dépassée. Si l'hétérogénéité de l'univers documentaire numérique, ou mieux la complexité croissante des univers numériques, n'est pas une donnée de base à partir de laquelle il faut construire, plutôt que de lutter vainement pour la réduire.

Comme les autres, et peut-être davantage, la profession des archivistes se projette dans l'avenir à la mesure de son enracinement dans le passé. Les objectifs qu'elle se fixe, les moyens qu'elle se donne, ou qu'elle aimerait qu'on lui fournisse, les critères d'évaluation avec lesquels elle se juge, elle se les représente longtemps à l'avance, au risque de s'y attacher trop exclusivement alors même qu'ils ne sont plus adéquats. La science des épigraphistes, paléographes et diplomatistes avait permis la reconquête de civilisations englouties. Des méthodes analogues et aussi rigoureuses n'auraient-elles pas, symétriquement, le pouvoir de nous conserver la mainmise sur les productions documentaires de la cybersociété ? A voir, mais à condition, assurément, de prendre du recul, de s'ouvrir aux autres disciplines et aux autres expérimentations, de se remettre en cause et de s'enrichir mutuellement. Accoler le qualificatif de numérique à celui de dépôt ne saurait résulter d'un simple tour de passe-passe, d'un effort paresseux de créativité. L'expression vient d'apparaître dans la littérature professionnelle. Que révèle-t-elle? Que cache-t-elle? Que nous promet-elle?

\section{Retrouver un équilibre compromis ?}

La tentation a donc été grande, et elle le reste, de rechercher en priorité la restauration d'un équilibre compromis : celui de l'organisation même du système d'archivage global que la civilisation industrielle s'était forgé au cours des deux derniers siècles, et dont le modèle fondamental reste auréolé d'une sorte de caractère universel ${ }^{1}$. Comment ne pas voir, dans le document numérique, le grain de sable

1. Le caractère «scientifique» de l'archivistique et de l'organisation des archives a pratiquement réussi à masquer les aspects politiques, au sens noble, de cette organisation. Au point que les mêmes références fondamentales semblent faire l'unanimité, dans la plupart des 
venu dérégler la belle mécanique ? Une fois surmontés les retards et les hésitations, bien explicables, voire nécessaires, avant de mettre au point «la» solution technique adéquate, tout ne serait plus qu'une question de moyens et de volonté politique. Est-ce aussi simple?

\subsection{Les documents numériques ne sont-ils qu'une catégorie de documents parmi d'autres?}

L'essentiel de la littérature consacrée jusqu'à maintenant aux documents numériques, quelle que soit la définition qui en a été donnée par les auteurs, a naturellement insisté sur la spécificité de ces documents par rapport aux documents dits traditionnels, c'est-à-dire aux documents enregistrés sur un support lisible directement par l'utilisateur'. Cette spécificité procède d'une évidence matérielle : c'est la nécessité, pour prendre connaissance de l'information, de recourir à une «machine» informatique (ordinateur et logiciel) capable de décoder l'information et de l'afficher sur un écran ou de l'imprimer. C'est d'ailleurs l'acronyme DLM (données lisibles par machine) qui a été retenu, au niveau européen, pour désigner ces documents numériques. En anglais, ce sont les adjectifs digital ou electronic, accolés à divers substantifs comme archive(s), data, document(s), information ou records qui les signalent parmi la diversité des supports.

Cette spécificité, conséquence inéluctable de l'évolution technologique dans les modes de production des documents, a eu pour effet immédiat de remettre en question les modes antérieurs de conservation et réutilisation de ces documents, en dehors et au-delà de leur environnement de production. Aussi la première question qui s'est imposée à tous les gestionnaires et utilisateurs potentiels des documents numériques a-t-elle été de savoir comment les sauvegarder, et surtout comment les relire, dans un autre environnement et dans un avenir plus lointain. Autrement dit, comment maintenir une continuité que le progrès technique venait perturber, alors qu'il est généralement considéré comme porteur de solutions plus efficaces et pertinentes.

Les réponses ont d'abord été recherchées dans l'harmonisation des standards, des normes, des règles et procédures de production et traitement documentaire, dans des guides de bonne pratique avalisés par l'ensemble de la profession, dans la sensibilisation des fournisseurs de machines, dans la prise en compte du problème par les législations et les programmes des gouvernements, dans la formation des

pays du monde, et qu'au sein d'un même pays elles n'entretiennent aucun clivage apparent entre les orientations des diverses formations politiques.

2. Il s'agit le plus souvent des documents sur support papier ou équivalent (parchemin ou toute autre surface inscriptible), lisibles «à l'œil nu» par quiconque est capable d'en comprendre la langage, mais les enregistrements de type analogique, lisibles avec un outillage simple restituant des images de ces documents, comme par exemple les microfilms, sont assimilables à ces documents traditionnels. 
utilisateurs. Une quête tous azimuts, dans l'espoir d'éviter que la majeure partie des informations et connaissances véhiculées dans ces documents numériques, omniprésents, ne tombent à jamais dans l'oubli, rendant notre société de plus en plus amnésique. Mais il est aujourd'hui légitime de se demander si cet effort, dont la nécessité n'est pas contestable, a été ou sera suffisant pour que les buts fixés puissent être atteints.

\subsection{Les limites d'une approche purement documentaire}

Toute cette recherche suppose implicitement que la découverte de solutions techniques harmonisées, leur mise sur le marché dans de bonnes conditions, leur prise en compte par les instances et procédures de régulation et les programmes de formation des utilisateurs auraient pour effet de rétablir quasi mécaniquement un équilibre de plus en plus malmené. Or cela n'est pas du tout certain. Il est même probable, même si elle se déplace, que la ligne de fracture ira en s'élargissant. Sauf à poser le problème en d'autres termes.

Le deuxième DLM-Forum ${ }^{3}$ d'octobre 1999 avait adressé un Message à l'industrie des technologies d'information et de communication. L'idée principale contenue dans ce message est celle d'un partage des tâches entre les professionnels de l'information d'une part, l'industrie des TIC de l'autre. Aux premiers, qui travaillent pour la plupart dans les institutions chargées de la conservation de la mémoire de la société et de sa mise à disposition des citoyens, il reviendrait de définir les besoins, « leurs propres besoins » pour être précis [INS 99]. A la seconde de proposer des solutions.

Dans sa réponse, publiée dans le numéro d'INSAR daté de l'automne 2000, l'industrie commence, à juste titre, - réponse du berger à la bergère ? - par demander au secteur public «de collecter, de mettre ensemble et d'harmoniser les demandes de solutions »[INS 00] et suggère à la Commission européenne de prendre un certain nombre d'initiatives concrètes pour permettre aux représentants du secteur public, et à ceux des citoyens, d'unir leurs efforts à ceux des industriels. Cela révèle la nature profonde du problème posé, qui n'est pas, d'abord, un problème technique. Si cela était, sans doute suffirait-il, faute d'un consensus préalable, de laisser s'imposer avec le temps quelques standards de fait, quitte à les officialiser après un toilettage de forme.

Or, ce que l'on doit constater aujourd'hui, c'est que la forme numérique est devenue la forme banalisée de la production comme de la diffusion, de l'échange et de la consultation des documents. Mais ce n'est pas une forme « unique », comme la

3. Réuni à Bruxelles, les 18 et 19 octobre 1999, à l'initiative de la Commission européenne, sur le thème : «Le citoyen européen et l'information électronique : la mémoire de la société de l'information». 
fameuse pensée du même nom. Ou alors, ce qui reste commun à l'ensemble de cette production aussi énorme que variée, ce sont des évidences contre lesquelles le bon sens industriel ne viendra pas s'insurger. Les acheteurs doivent pouvoir lire, écrire, écouter, visionner, relire avec le matériel dont ils disposent pendant un minimum de temps : celui de la durée de vie de ce matériel lui-même. Et quand ce matériel évolue, le transfert des données doit être réalisable à un prix acceptable. Ces dernières années, ce processus a pu s'observer aussi bien dans l'évolution des systèmes d'information des grandes administrations et entreprises, que dans celle de marchés dits «grand public » tels que ceux des phonogrammes, des vidéogrammes ou de la photographie familiale. Qu'on le veuille ou non, les arbitrages techniques en matière de normes, standards et procédures se feront entre les fournisseurs de solutions d'une part, et les maitres d'ouvrages ou clients individuels qui les financent d'autre part, en fonction de leurs besoins propres, calculés au plus juste.

\subsection{Vers une approche « systémique»?}

Les exigences des organismes en charge de la conservation ultime des informations auront peu de poids si elles restent aux bornes de leur propre organisation interne. Alors que ces problèmes étaient naguère dissociés, dans le temps et dans l'espace, il est essentiel aujourd'hui de les aborder en prenant en compte, en même temps, les contraintes des producteurs et celles des utilisateurs de ces informations archivables. Toutes les préconisations justifiées par la garantie de la fiabilité et de l'authenticité des données, pendant toute la durée de temps requise, dans l'intérêt supérieur des citoyens, en vue de la préservation de leurs droits et de leur sécurité individuelle et collective, devraient être facilement acceptées. Il subsiste alors un problème technique: celui de la traduction des garanties demandées en cahiers des charges réalistes. Mais il devient secondaire. Il peut se traiter par étapes ou par domaines, en fonction des urgences et priorités. Le politique reprend en main la conduite du processus. Le mythe de la solution technique universelle applicable a priori à l'ensemble des documents numériques est écarté. Le débat s'en trouve clarifié.

Le gestionnaire de documents ou l'archiviste qui se voulait "généraliste», l'institution qui avait la mission de conserver la mémoire de toute une collectivité, indépendamment de la nature physique des supports, après une sélection portant sur la valeur intrinsèque des informations et non pas sur sa capacité technique à manipuler et exploiter les documents, doivent-ils alors remettre en cause toute la structure technique et mentale sur laquelle ils s'appuyaient ? Ce serait évidemment tomber dans l'excès inverse, encore plus détestable. On ne quitte pas un appartement devenu trop étroit avant d'avoir trouvé un logement plus spacieux. Et il ne s'agit pas ici d'abandonner subitement l'utopie de règles universelles pour le traitement des documents numériques. Cette utopie peut rester féconde sur le plan intellectuel, elle peut être un garde-fou salutaire contre une tendance à l'émiettement excessif des 
connaissances. Il s'agit, plus pragmatiquement, plus tactiquement, de ne pas mettre tout de suite la barre trop haut. Et d'ailleurs, à regarder les choses du dehors, ce sont bien de grands ensembles perméables mais distincts, aux frontières fluctuantes mais jaloux de leur propre autonomie, qui se partagent la gestion des supports de la connaissance; que la distinction entre les uns et les autres soit fondée sur des divisions territoriales, sur des concepts idéologiques (le public et le privé, par exemple), ou sur des «natures» de documents (archives, bibliothèques, phonothèques, vidéothèques, banques de données...), qui ne manquent pas d'évoluer avec le temps. Or, des efforts substantiels qui sont faits ici et là pour numériser les documents produits antérieurement, essentiellement pour d'excellentes raisons de sécurité des originaux ou de plus grande facilité d'accès au plus grand nombre d'utilisateurs intéressés, retire-t-on l'idée qu'il faudrait regrouper ensemble les institutions qui vont conserver sur des supports numériques techniquement identiques des images de documents antérieurs dont la conservation des originaux relèveraient plutôt des uns ou des autres, selon la nature ou la provenance de ces originaux?

Les institutions traditionnelles vouées à la gestion des archives se trouvent donc à la croisée des chemins. Soit elles se recentreront sur leur rôle de conservatoires d'antiquités, se préoccupant en priorité des documents dits historiques. Elles se spécialiseront de ce fait dans un sous-système particulier du système d'information global de la société. Alors, elles laisseront un espace vacant qui devra se remplir d'une manière ou d'une autre. Soit, sans pour autant leur faire abandonner leurs missions patrimoniales, de nouvelles missions leur seront confiées pour remplir ce vide. Nouvelles par leurs caractéristiques du moment, mais fidèles à l'une des missions premières de ces institutions depuis qu'elles existent. Leur histoire nous montre en effet que c'est d'abord pour satisfaire des besoins liés à une gestion plus efficace d'une société en évolution, des besoins patrimoniaux au sens domanial, que des «Archives» ont été constituées, avant même qu'elles ne développent leur dimension historique, patrimoniale au sens culturel ${ }^{4}$.

\section{L'émergence d'un nouveau concept : le dépôt numérique ?}

Nous tiendrons donc pour acquis, à ce stade de notre réflexion, et sous réserve de le remettre en cause ultérieurement si des arguments pertinents et motivés l'exigeaient, que le concept de "document numérique », avec tout son cortège : définition(s), normes de production, règles de conservation et d'exploitation, méthodes d'analyse et de traitement..., ne peut, à lui seul, résoudre les problèmes

4. La tradition archivistique française fait remonter l'origine des Archives de France à la bataille de Fréteval. Philippe-Auguste, ayant abandonné à l'ennemi les titres qui jusqu'alors accompagnaient la cour du roi dans son nomadisme, décida, pour des raisons évidentes de sécurité, de conserver dorénavant le trésor de ses chartes dans la tour principale de la forteresse du Louvre qu'il fit construire à Paris, sa capitale. 
auxquels sont aujourd'hui confrontés les professionnels et les institutions qui ont la charge de conserver ces documents pour le compte de la collectivité. Cela ne remet nullement en cause tout le travail déjà accompli, ni celui qui reste à faire sur ce point particulier. Il s'agit d'ouvrir une nouvelle piste à explorer, en complément, et à l'aide des résultats déjà obtenus par les études et recherches sur ce concept.

\subsection{La banalisation du «tout numérique »?}

On peut donc dire, aujourd'hui, que tout document d'archives est, a été ou sera numérique. Qu'il s'agisse de procédures administratives, d'échanges commerciaux, de diffusion d'informations auprès du grand public ou de correspondances privées entre personnes individuelles, la lettre manuscrite ou dactylographiée devient l'exception (ce qui est d'ailleurs loin de diminuer sa valeur) maintenant que les outils de production de documents numériques, textes comme images, sont répandus dans presque tous les bureaux et presque tous les foyers.

Quant aux documents, produits antérieurement, que leur intérêt ou le hasard nous ont conservés, la prolongation même de cette conservation passe aujourd'hui le plus souvent, depuis la vénérable et prestigieuse institution jusqu'au moindre bureau d'adolescent équipé d'un scanner, par la fabrication de ces «clones numériques » qui nous libèrent du risque du prêt, qui nous donnent cette joie de pouvoir partager largement sans se démunir.

Et ce qui est vrai pour les documents pris isolément l'est aussi pour les ensembles qu'ils composent, quelle que soit leur nature ou leur dimensions : collections, séries, fonds... Il est donc tout à fait normal que la plupart des archivistes puissent s'imaginer qu'une fois les adaptations technologiques réalisées (ce qui est déjà, en soi, un très vaste programme), tout dépôt d'archives sera devenu, de facto, un dépôt numérique, c'est-à-dire un organe capable de collecter, traiter, conserver, restituer aux utilisateurs tous types de document numérique ayant dépassé le stade d'utilité courante de son cycle de vie (sans avoir reposé la question du transfert du document entre producteur et archiviste, de son objet et de sa justification).

Tout doit-il donc se passer comme si le principal problème à résoudre n'était en fait qu'un problème d'adaptation et de mise à niveau technologique, donc un problème de moyens nouveaux, matériels et humains, donc financiers, à dégager le plus vite possible, faute de quoi surviendrait la catastrophe annoncée ? Ne serait-ce pas tomber dans le même travers que précédemment, quand nous parlions « du » document numérique, mais en raisonnant cette fois au niveau d'un ensemble structuré de documents numériques, regroupés sur le seul critère de leur acquisition par un dépôt d'archives traditionnel, devenu « dépôt numérique » parce que doté des moyens techniques pour recevoir, conserver et communiquer des documents 
numériques? Quand l'électricité est venue améliorer considérablement les conditions de conservation des vieux dépôts froids, sombres et humides, les « dépôts électriques » ont-ils changé quelque chose de fondamental dans l'organisation des archives?

\subsection{Qu'est-ce qu'un dépôt numérique?}

Si l'on s'en tient à cette première approche, tout à fait naturelle et légitime, étape nécessaire dans le débat et la réflexion, je crains que l'on ne prenne un double risque. Le risque de s'engager dans une course-poursuite épuisante et décevante, et le risque de passer à côté d'une autre approche, peut-être plus prometteuse. Je dis «peut-être » car je n'en suis encore qu'au stade des intuitions, pas à celui des déductions et solutions démontrables. Cet article doit être compris comme une première contribution dans une réflexion à poursuivre. A ce stade, la meilleure contribution ne peut donc pas être une démonstration. Je préfère vous faire partager les raisons de mes interrogations.

Celles-ci sont nées et se sont peu à peu nourries dans la lecture concomitante et comparative des actes des deux DLM-Forums de 1996 et 1999. Ma dette est donc très grande à l'égard de celles et ceux qui ont apporté leurs contributions à ces journées de réflexion, autant qu'à celles et ceux qui ont permis leur rapide publication. Après, je dois l'avouer, une première impression de déjà vu, au-delà des informations ponctuelles toujours très enrichissantes relatives à des projets particuliers, ce qui est une illusion facile pour un professionnel amené à lire assez régulièrement et depuis longtemps de (trop ?) nombreuses publications sur le sujet, j'ai bénéficié d'une chance : un changement presque total de préoccupations pendant près d'un an. Mais une relecture après cette jachère m'a fait brusquement apparaître, comme une sorte d'évidence, quelques constatations qui peuvent être, aujourd'hui, au cœur de la réflexion.

Alors que les communications du DLM-Forum de 1996 étaient centrées quasi exclusivement, c'était la moindre des choses, sur les problèmes de conservation et traitement des données et documents électroniques, j’ai été frappé, à la lecture des actes du DLM Forum de 1999, par l'apparition, discrète mais répétée, de l'expression de «dépôt numérique » (en anglais : digital or electronic repository). S'agit-il déjà d'un nouveau concept formalisé ? Probablement pas encore. Je n'en ai pas trouvé de définition développée et argumentée (ai-je bien tout lu ?). Mais j’ai le sentiment qu'il s'agit d'autre chose que des commodités du langage courant ou des hasards de la traduction simultanée, qu'il y a là comme une sorte d'embryon de concept, dont la survie n'est pas assurée, mais qui peut aussi être souche d'une postérité féconde. C'est en cherchant à en savoir plus que j'ai commencé à mettre bout à bout les premières réflexions que je livre dans cet article. 
Je m'en tiendrai ici à trois citations. La première est tirée de la préface des actes de 1999 [INS 99] qui renvoie au discours de M. Erkki Liikanen, Commissaire européen pour l'entreprise et la société de l'information. Elle évoque les dépôts d'informations électroniques (ou numériques) comme les lieux de conservation d'un immense potentiel de connaissances qu'il convient de préserver et de rendre accessible, ce qui devrait susciter l'intérêt de l'industrie en jetant les bases d'un nouveau partenariat entre les secteurs public et privé. La deuxième concerne le projet hollandais de « Digital Repository ». La troisième renvoie à la communication de M. Gerhard Wagner citant lui-même M. Parajon Collada en posant la question : «What is a digital library ${ }^{5}$ ?».

Et je retire du reste de ma lecture des actes de 1999, complétée par une navigation sur l'internet avec le mot-clé «digital repository», les constatations suivantes :

- l'expression digital repository, que je traduis, faute de mieux, par « dépôt numérique », existe, même si l'expression «sœur » de digital library est beaucoup plus répandue ;

- elle recouvre des réalités assez diverses qui ont cependant un point commun : conserver et mettre à la disposition d'usagers, sous une forme numérique, un ensemble structuré d'informations couvrant un domaine bien délimité du patrimoine des connaissances de l'humanité ;

- elle intéresse aussi bien des données et connaissances du domaine culturel que de l'activité économique et sociale la plus contemporaine ${ }^{6}$;

- chacune a sa vocation ou sa mission spécifique, délimitée, condition indispensable pour être une référence dans son domaine, mais elle n'est plus limitée

5. [INS 99]. La préface est signée par Mme Sarah Tyacke, Keeper of Public Records (GrandeBretagne), et par M. Hans Hofmann, Head of the Historical Archives of the European Commission. "He noted that the preservation and accessibility of the immense potential contained in electronic information repositories could provide a broad area of service that should wake the interest of industry and serve as the basis for a new partnership between public and private sectors $»$.

Plus loin, Johannes Hofman parle de «Shooting at a moving target. The development of a repository for the preservation of digital information ».

Enfin (p. 152) il est rapporté que «...Mr Parajon Collada, Deputy Director-General for the Information Society, yesterday said the following, and I should like to quote him : What is a digital library? A common definition is lacking. We need new terminologies to shape our actions. »

6. [INS 99], p. 196. Maike Bielfeldt, «Pilot projects in Germany and Europe for the improvement of the application of the digital signature ». Projet très intéressant conduit par l'association nationale des Chambres de commerce et d'industrie allemandes, et ses correspondantes à l'étranger afin de disposer d'un système fiable et performant pour gérer les contrats de formation professionnelle et les certificats d'origine des produits exportés. 
par les contraintes d'ordre territorial, ou de nature ou provenance des originaux, qui prévalaient avant la duplication illimitée des images permise par le numérique ;

- s'y substituent, ou devront s'y substituer, des règles particulières de gestion des droits d'accès, de garantie de la fiabilité et de l'authenticité des informations accessibles, de sécurité et de protection des données conservées et des droits des personnes concernées ;

- le besoin d'une définition claire de ce concept est explicite, ainsi que de codes de bonne conduite ;

- les dépôts d'archives publiques traditionnels ont vocation, avec bien d'autres organismes, publics ou privés - au premier rang desquels se trouvent les bibliothèques - à faire partie de ces « dépôts numériques »;

- une bonne part des problèmes qu'ils ont à résoudre peuvent et doivent l'être au niveau de cet ensemble beaucoup plus large constitué par l'ensemble de tous les dépôts numériques actuels et à venir («Dépôts numériques de tous pays, unissezvous !»);

- sans négliger les questions de pure technologie, il est évident que ces principaux problèmes à résoudre sont d'ordre éthique ou moral, juridique, organisationnel, politique au sens large et noble du terme.

C'est en essayant de dégager quelques conséquences possibles de l'adaptation de ce nouveau concept à l'organisation archivistique que je terminerai ce papier, en insistant encore sur le caractère exploratoire et inachevé de cette réflexion.

\section{Conséquences sur l'organisation archivistique}

Les deux axes que j'ai retenus, sur lesquels j'entrevois des possibilités d'évolutions intéressantes, l'ont été pour deux raisons : premièrement, j'y avais été sensibilisé par mes expériences professionnelles des cinq dernières années et j'ai retrouvé des résonances avec ces expériences à la lecture des actes des DLM Forums; deuxièmement, ces deux axes, confrontés à mon expérience antérieure d'archiviste public pendant une quinzaine d'années, me semblent susceptibles d'apporter des éléments constructifs à la «crise » que l'on dit secouer aujourd'hui cette profession?

Le premier de ces axes est relatif aux brèches, mais aussi aux complémentarités, que le numérique ouvrira dans l'organisation territoriale traditionnelle des archives. Le second concerne des évolutions analogues sur une autre «frontière», celle qui est censée séparer secteur public et secteur privé.

7. Cf. les publications récentes de l'Association des Archivistes français et la création, le 17 janvier 2001, de l'association « Une cité pour les Archives nationales ». 


\subsection{De l'éclatement des frontières territoriales sous l'impact du numérique}

Dans une société de l'information globale, où tant de données et de connaissances peuvent tout à la fois menacer ou sauver des vies humaines, le rôle des archivistes soucieux du bien public ne peut que se développer s'ils savent ne pas se cantonner au domaine éminent, mais limité, des archives historiques. S'ils osent investir tous les champs d'informations socialement utiles à leurs concitoyens, en vue de leur garantir la fiabilité et l'authenticité de ces informations. C'est en effet à travers un réseau de dépôts numériques, transcendant les anciennes frontières territoriales, que les citoyens du monde du XXI ${ }^{\mathrm{e}}$ siècle, à commencer par les citoyens de l'Union européenne, pourront voir conservées, avec toutes les garanties requises en matière de confidentialité et d'authenticité, les données fondamentales qui devront être conservées fidèlement sur de longues périodes, mais auxquelles ils devront pouvoir accéder dans des délais parfois très brefs, en toute sécurité.

La liste des domaines d'application possible est très vaste. Pour ne donner que quelques exemples, à titre indicatif, on peut citer le domaine de la santé, celui de la sécurité alimentaire, celui de la protection des droits et des biens individuels. Pour pouvoir se faire soigner dans des conditions optimum, dans le plus grand nombre possible de régions du monde, il ne suffira pas d'une carte à puce que l'on peut égarer: une banque de données centrale, correctement sécurisée et contrôlée, indépendante des autorités politiques, en est le complément indispensable. Pour suivre et «tracer» les informations concernant les produits alimentaires, qui franchissent de nombreuses frontières avant d'être consommés, une instance internationale, indépendante mais restant sous contrôle des citoyens, doit pouvoir assurer les échanges d'informations indispensables entre producteurs, commerçants, consommateurs et pouvoirs publics. En matière de protection des droits, d'assurance des biens, d'évaluation des préjudices subis, on imagine facilement tout ce que pourraient apporter des institutions débordant les frontières territoriales.

Des exemples existent dans notre histoire de telles institutions d'intérêt commun qui ne sont pas pour autant des administrations de l'Etat, à commencer, dans les pays où elle existe encore et rend de grands services, par l'institution du notariat. Sans forcément le recopier servilement, c'est un exemple dont on peut s'inspirer, en se souvenant qu'une part non négligeable de la responsabilité des notaires, celle qui consiste en la garde des minutes, est, fonctionnellement parlant, une mission d'archiviste.

\subsection{De la nécessaire coopération entre secteurs public et privé}

Il fut un temps où la puissance publique, en dehors de sa propension à vouloir contrôler « l'esprit public » et tout de système de diffusion et propagation des idées à travers les documents imprimés, pouvait s'imaginer faire mieux respecter ses 
prérogatives en se réservant ou en octroyant sélectivement des privilèges d'utilisation de tel ou tel mode d'expression et de diffusion des idées. Les diplômes, les bulles ou les chartes des grands de ce monde étaient sans conteste les documents les plus beaux et les mieux faits de leur temps. Les documents privés n'en étaient que de pâles et respectueuses imitations. La fabrication d'un faux billet était passible d'une peine bien supérieure à ce qu'aurait mérité le seul préjudice financier réellement causé.

Dans notre monde d'aujourd'hui, c'est plutôt la société dite civile qui donne le la dans ce qu'on pourrait appeler la «mode documentaire ». Le terme « officiel» en gros et gras caractères n'apparaît plus guère que sur des prospectus publicitaires banalisés. Les collectivités publiques abandonnent leurs anciennes «armes » pour des logos qui les mettent au rang des firmes et sociétés ordinaires. Les austères journaux ou bulletins officiels ne sont plus guère conçus pour être lus, et sont remplacés par des publications dont la forme et le style extérieur sont empruntés aux publications commerciales.

Mais la quasi-disparition d'une hiérarchie fondée sur la sacralisation du pouvoir, réservant aux gouvernants le contrôle et la maîtrise de l'information, n'est pas le seul élément à prendre en compte. Dans les trois exemples que nous venons de prendre de possibles réseaux de dépôts numériques transfrontaliers au service des citoyens, nous pouvons constater qu'à chaque fois, nous avons trois interlocuteurs en présence : les citoyens, bénéficiaires ultimes ; les fournisseurs d'informations que sont les principaux acteurs de la vie économique et sociale : professionnels de santé, industrie pharmaceutique, agriculteurs, industrie agroalimentaire, professionnels de la distribution, assureurs, etc. ; la puissance publique chargée du contrôle et de la régulation, d'autant plus efficace et crédible qu'elle ne sera pas juge et partie.

\subsection{De l'évolution des autres principes de l'archivistique}

Outre le débordement des frontières territoriales et le dépassement d'un cloisonnement trop rigide entre public et privé, l'impact des technologies numériques vient bouleverser assez fortement la répartition des tâches et l'économie du système, s'il ne remet pas nécessairement en cause (ce serait quand même à bien examiner) les principes de base de la gestion des documents d'archives ou du records management (respect du cycle de vie des documents, respect des fonds et principe de provenance).

L'effet le plus apparent de cet impact va consister dans la disparition progressive de l'acte archivistique majeur du système traditionnel, le versement, qui symbolisait et matérialisait le transfert de compétence entre producteurs de documents et archivistes, quand on ne pouvait pas faire autrement que de loger les documents d'archives en un lieu unique. Aujourd'hui, non seulement la duplication est 
techniquement possible, mais elle est souvent souhaitable pour des raisons de sécurité. Producteurs d'archives et archivistes doivent donc repenser complètement leurs modes de fonctionnement.

Il n'est pas possible de décrire avec précision une aussi vaste mutation en quelques lignes. Disons simplement, pour ouvrir quelques pistes de réflexion, que cette mutation sera progressive, mais profonde, et qu'elle verra des archivistes venir travailler dans l'environnement immédiat des producteurs, pas nécessairement de manière permanente, mais selon des cycles périodiques de collaboration qui sont à inventer. D'un autre côté, on verra certainement les dépôts d'archives traditionnels, dotés des équipements technologiques adaptés, ce que nous appelons ici les dépôts numériques, abandonner peu à peu leur caractère généraliste pour se spécialiser sur les créneaux archivistiques pour lesquels ils auront développé un savoir-faire reconnu. Ce que les archivistes ont jusqu'à présent appelé "l'archivistique spéciale $^{8}$ » est ainsi très probablement destinée à un remarquable développement dans les décennies à venir. Cela ne manquera pas de susciter, sinon une concurrence, du moins une certaine émulation dans la profession, ce qui ne sera pas le moindre des changements à faire comprendre et accepter.

\section{Conclusion}

Le phénomène le plus frappant qui aura caractérisé le $\mathrm{xx}^{\mathrm{e}}$ siècle dans le domaine de l'archivistique est sans conteste l'accroissement toujours plus accéléré de la masse des papiers et autres supports d'information. Bien des archivistes se seront essoufflés à tenter de rattraper et de canaliser cette production galopante. Jamais les dépôts d'archives n'auront été aussi spacieux, jamais les archivistes n'auront été aussi nombreux à sentir leur proie leur échapper, sauf à crier «Stop ", à poser des bornes sur le chemin de l'histoire et à limiter leurs perspectives au traitement des fonds clos.

Le $\mathrm{XXI}^{\mathrm{e}}$ siècle commence dans un nouveau décor. L'ancienne problématique est maintenant obsolète. Les technologies du numérique ont changé la donne. La place pour engranger n'est plus le besoin prioritaire, les tris et éliminations se posent en termes nouveaux. Ce qui importe maintenant, c'est l'art de la navigation sur des océans et dans des espaces en fulgurante expansion. Car il est vain de vouloir arrêter la course du monde, de dresser des digues, d'imposer des limites aux besoins que la vie des hommes d'aujourd'hui rend indispensables à la survie des hommes de demain.

8. L'archivistique spéciale traite des problèmes de traitement, conservation et exploitation propres à des ensembles archivistiques délimités comme tels : minutes notariales, archives d'architecture, archives de laboratoires, etc. 
A la fin du $\mathrm{Xx}^{\mathrm{e}}$ siècle, dans le monde dit développé, il était quasiment devenu impossible de survivre «sans papiers ». La parole humaine n'était plus suffisante pour justifier le moindre droit, à commencer par celui de vivre. Au XXI siècle, dans ce même monde condamné à se développer toujours plus, sauf à régresser brutalement dans la barbarie, il faudra toujours plus et constamment démontrer qui l'on est, d'où l'on vient, quels sont ses droits, si l'on est autorisé à savoir ci, à se rendre là, à vendre ceci, à consommer cela. Il faudra prouver d'où vient son lait, sa viande, son eau, son sang, ses sources... Oh ! rassurez-vous, plus besoin de papiers, que l'on perd et qu'il faut refaire en présentant d'autres papiers qui eux-mêmes... Quelques cartes, quelques puces, quelques codes émis à partir d'implants scellés au plus profond de votre corps veilleront sur vous comme autant d'anges gardiens. Un réseau mondial de dépôts d'archives numériques, surveillés et contrôlés plus sévèrement qu'aucune centrale nucléaire du siècle passé, servis par des légions d'archivistes insoupçonnables et assermentés, entretiendra avec vos T3P (terminaux personnels portables permanents) un dialogue constant, en temps réel ou différé, selon l'urgence des besoins et les nécessités de la régulation des trafics. Qui vous êtes, qui sont vos interlocuteurs, vos amis, votre prochain, et même vos ennemis, ce que vous mangez, buvez, respirez, exhalez, proférez ou professez, tout sera instantanément analysé, vérifié, mis à jour, recoupé, validé, authentifié. Toute anomalie sera aussitôt signalée par un bip sonore, un voyant lumineux, ou un signal plus discret selon les options de votre contrat. Rassurez-vous encore : ce que vous pensez restera votre domaine réservé, inaliénable, inviolable, selon les termes même d'une charte solennellement ratifiée à l'unanimité par la communauté internationale. Et tout manquement à ce code de bonne conduite immanquablement déclaré comme intolérable et inacceptable par les plus hautes autorités sur l'ensemble des médias... Que les archivistes, eux aussi, se rassurent: un champ immense s'ouvre pour l'exercice rénové de leur responsabilité sociale de médiateurs de l'information authentique.

\section{Bibliographie}

[INS 96] INSAR (INformation Summary on ARchives - Courrier européen des archives), Supplément II (1997): Actes du DLM-Forum sur les données lisibles par machine, Bruxelles, 18-20 décembre 1996, édité par l'Office des publications officielles des communautés européennes, L-2985 Luxembourg.

[INS 99] INSAR, Supplément IV (2000), Proceedings on the DLM-Forum on the electronics records, Brussels, 18-19 October 1999, 359 p.

[INS 00] INSAR, n8 8, automne 2000 (archis@ cec.eu.int). 